\title{
The Flavr Savr Tomato, an Early Example of RNAi Technology
}

\author{
Elysia K. Krieger ${ }^{1}$, Edwards Allen, Larry A. Gilbertson, \\ and James K. Roberts
}

Monsanto Company, Chesterfield Campus, 700 Chesterfield Parkway, Chesterfield, MO 63017

\author{
William Hiatt and Rick A. Sanders \\ Monsanto Company, Calgene Campus, 1920 5th Street, Davis, CA 95616
}

Additional index words. Flavr Savr tomato, polygalacturonase, RNAi, T-DNA linkage

\begin{abstract}
The Flavr Savr tomato was introduced as the first genetically engineered whole food in 1994. The commercial event, resulting from transformation with an antisense expression cassette of the endogenous polygalacturonase gene, was sequenced and found to contain two contiguous, linked, transfer DNA insertions. We found polygalacturonase suppression correlates with accumulation of $\approx 21$-nt small interfering RNAs, the hallmark of an RNA interference-mediated suppression mechanism.
\end{abstract}

The Flavr Savr tomato has increased storage life through suppression of the tomato polygalacturonase $(P G)$ gene, resulting from transformation of an antisense expression cassette of the $P G$ cDNA (pCGN1436) (Sheehy et al., 1988). Efficacious but noncommercial pCGN1436 transformation events contain multiple T-DNA insertions linked by right borders (Redenbaugh et al., 1992; Sanders and Hiatt, 2005), leading to the hypothesis that readthrough transcription of the $P G$-linked antisense (PGAS) cassettes resulted in RNA interference (RNAi)-mediated PG suppression, rather than antisensemediated sequestration, of the endogenous $P G$ transcript (Watson et al., 2005). To test this hypothesis, we determined if RNAi suppression is responsible for the Flavr Savr phenotype by characterizing the T-DNA locus of the Flavr Savr tomato event and analyzing for the presence of 21-nt PG small interfering RNAs (siRNAs) diagnostic of RNAi.

\section{Materials and Methods}

Tomato plants. CF5013 is the tomato cultivar that resulted from a nonconventional cross between the homozygous PGAS inbred line CR3-1436-613:@.12 with the tomato hybrid Mountain Spring. CF5013 is heterozygous for the PGAS gene which is dominant and was sold commercially as the Flavr Savr tomato. These plants were selfed, and resulting sibling plants were screened by taqman PCR for NptII. Of the 64 plants screened, the ratio was 43 to $21 \mathrm{NptII}$ positive to negative plants, which is consistent with a $3: 1$ ratio of transgenic to non-transgenic tomato plants $\left(\chi^{2} P=0.15\right)$.

Received for publication 23 Dec. 2007. Accepted for publication 6 Jan. 2008.

We thank Daniel Free and Christina Kavanaugh for greenhouse support.

${ }^{1}$ To whom reprint requests should be addressed; e-mail elysia.k.krieger@monsanto.com
Southern blots and inverse PCRs. Genomic DNA was isolated from tomato leaves, and EcoRV Southern blots were done according to Redenbaugh et al. (1992). To make inverse PCR libraries, $10 \mu \mathrm{g}$ of genomic DNA was digested separately with XhoI, NcoI, EcoRV, HindIII, and PstI and ligated to itself. Primers complementary to regions of the T-DNA were used to walk out across the T-DNA inserts as well as into genomic flanking regions. PCRs were done with Extaq (Takara Bio, Shiga, Japan), and products were cloned and sequenced using pCR2.1TOPO (Invitrogen Corp., Carlsbad, CA).

PCR primers.

1: TATACCCGCAGTCCGCTCACCCTACC 2: ACACGAAACTCATGAGGGAGGAGATG 3: GAGACCGATGTTCGTTCCGGAACCTT 4: AAGGTTCCGGAACGAACATCGGTCTC 5: ATTCAAAAGTCGTTAATGGCTGCGG ATCAAG

6: CAATTGTAAATGGCTTCATGTCCGG GAAATC

PG enzyme activity assays. PG enzyme activity assays, measured as an A540 absorbance, were done in replicates of six according to Redenbaugh et al. (1992). The average enzyme activity of the three transgenic tomatoes $(4523,3951,3949)$ was $0.058,0.073$, and 0.075 , respectively, compared with the nulls $(4193,4131,3975)$, which were 1.032 , 1.11 , and 0.98 .

RNA purification and Northern blots. Total RNA was purified from ripe tomatoes using Trizol (Invitrogen) and further cleaned using the RNA/DNA Midi Kit (Qiagen, Valencia, CA), according to procedures provided by the manufacturer. Low molecular weight Northern blots were done with 17\% polyacrylamide gels (Llave et al., 2002a), and high molecular weight Northern blots were done with $1.5 \%$ agarose gels (Llave et al., 2002b). Blots were hybridized with radioactive 42-nt isotopically labeled LNA oligo probes or DNA probes complementary to $P G$. DNA oligo probes were ordered from IDT; a "+" symbol preceding represents an LNA-modified nucleotide. Sequences used were as follows:

\section{A1: AAAC + ATA + TGA + TAATATT + GCA + TTT + GAGCAAG + CAT + GGA + ATGAA A2: ATTA+TAA+TGG+AGAATAT+AAA + \\ TTA + GTAGGGG+AAA + GTG+GAAAA B1: $\mathrm{ATGC}+\mathrm{AAT}+\mathrm{ATT}+\mathrm{ATCATAT}+\mathrm{GTT}+$ $\mathrm{TTT}+\mathrm{CCATCAC}+\mathrm{CCT}+\mathrm{TAG}+\mathrm{CTCCA}$ B2: ATAG+TCT+ATA+ATTATGG+GAT+ ACT+TAACGTC+TTG+CAT+TTCCA}

Oligos were isotopically labeled with $\left[\gamma-{ }^{32} \mathrm{P}\right]$ ATP as described in Allen et al. (2005). DNA probes: Probe C-First 483 bp of the NptII sequence. Probe D-Last 420 bp of the $P G$ sequence. DNA probes were labeled with dCTP using the RadPrime DNA-labeling system (catalog no. 18428011, Invitrogen) according to the manufacturer's directions.

\section{Results}

Commercial Flavr Savr locus contains two linked T-DNAs. Plants from commercial hybrid seed (CR3-1436-613:@.12/ CF5013), not included in the previous analysis (Sanders and Hiatt, 2005), were grown and selfed to obtain positive (4523, 3951, 3949) and null (4193, 4131, 3975) sibling plants for molecular analysis. Genomic DNA from these plants was digested with EcoRV, and Southern blot hybridization was performed to determine T-DNA copy number and linkage (Fig. 1A). Bands were observed in the transgenic plants of 3698- and 5162-bp corresponding to the $P G$ region of the locus, in addition to a $2-\mathrm{kb}$ band of the native $P G$ gene present in all lanes. This is consistent with the presence of two copies of the $P G$ region of the T-DNA. The transgene locus and flanking genomic sequence were obtained by inverse PCR amplification, cloning, and sequence analysis of resulting fragments. The locus sequence confirmed the T-DNA linkage at the left border (LB) of one of the two T-DNAs; the second T-DNA is truncated, removing all of the LB sequences and part of the MAS promoter (Fig. 1B). These results were further confirmed by amplifying the T-DNAs with flanking genomic and unique junction region primers (Fig. 1C) in transgenic (4523) and null (4193) siblings. The 6974-bp band (lane 1) contains the truncated (left) T-DNA. The 7725-bp band (lane 3) contains the full-length (right) T-DNA. We were unable to amplify across the insert (14,674-bp, lane 5), likely due to the inverted repeat sequence. In the null sibling, these primers amplify a 1058-bp band (lane 6) verifying insert location. The 764-bp band (lane 7) is the product across the unique T-DNA junction sequence. Taken together, these results show the commercial Flavr Savr tomato contained two T-DNAs linked in an inverted orientation. 


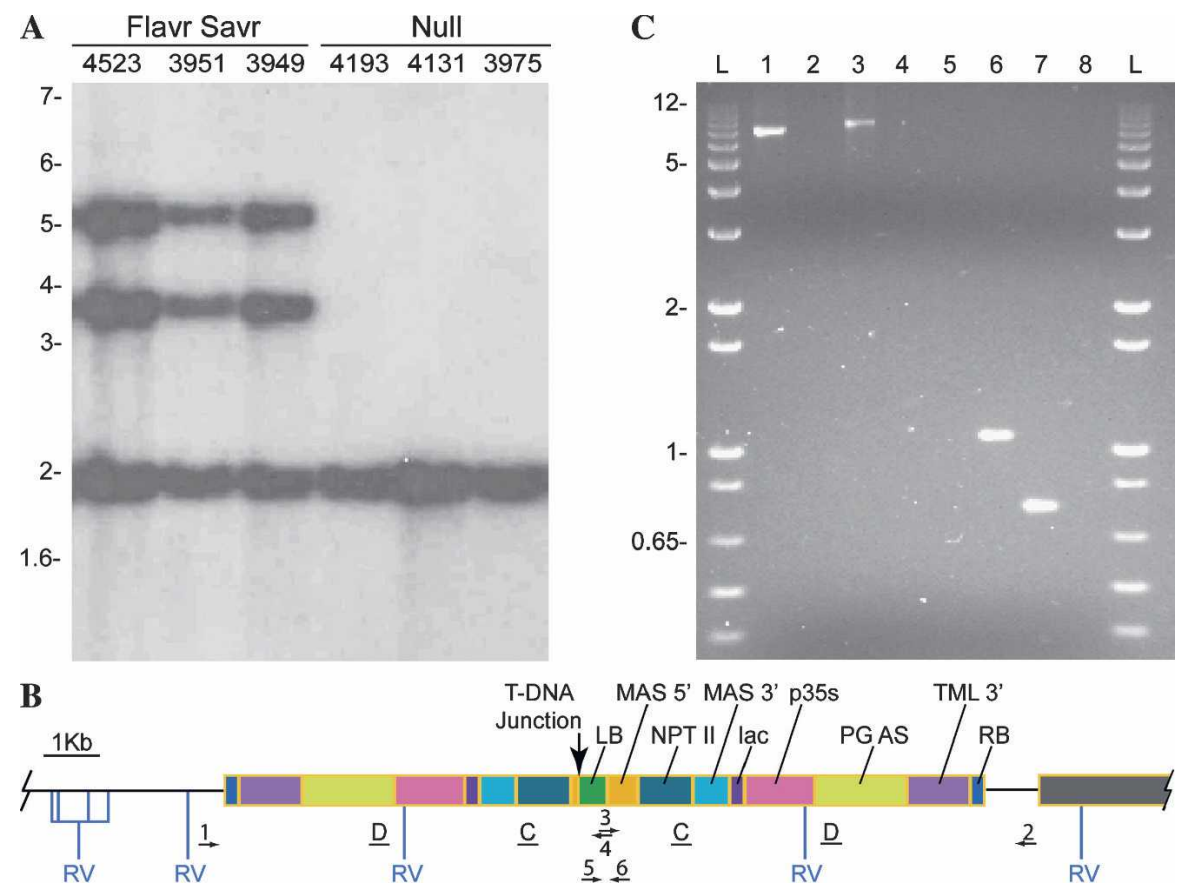

Fig. 1. Characterization of siblings of the Flavr Savr tomato. (A) Southern blot confirmation of T-DNA number using probe $\mathrm{D}$ of the $P G$ gene. Transgenic (4523, 3951, and 3949) and non-transgenic (4193, 4131, and 3975) siblings were analyzed. The T-DNA insertion corresponds to the 3.6- and 5.1-kb hybridizing bands. (B) Diagram of the sequenced 13,621-bp T-DNA insertion and flanking genomic regions. DNA probes (bars $\mathrm{C}$ and D) and primers (arrows 1-6) used for confirmation PCRs are indicated. A putative CYP82 family cytochrome P450 mono-oxygenase gene in the adjacent genomic sequence is indicated (gray box). (C) Confirmation of linked T-DNA orientation by PCR with genomic flank and unique junction sequence primers. PCRs are shown in pairs; odd-numbered lanes use transgenic sibling 4523 genomic DNA, even-numbered lanes use null sibling 4193 genomic DNA. Sizes of the marker bands (lane L) are indicated at right. Left T-DNA, lanes 1 and 2 (primers 1 and 4); right T-DNA, lanes 3 and 4 (primers 2 and 3); full insert, lanes 5 and 6 (primers 1 and 2); unique junction region, lanes 7 and 8 (primers 5 and 6 ).

siRNAs from transgenes accumulate in Flavr Savr tomatoes. To investigate if $\mathrm{PG}$ silencing is the result of an RNAi-mediated pathway, we confirmed the phenotype of the Flavr Savr tomato by PG enzyme activity assays (Redenbaugh et al., 1992) and assessed accumulation of $\mathrm{PG}$ mRNA and siRNAs. PG enzyme activity assays on ripe tomatoes using a polygalacturonic acid substrate (Redenbaugh et al., 1992) showed relative activity of $0.069 \pm 0.009$ (SD) in transgenic vs. $1.041 \pm 0.065(\mathrm{SD})$ in null siblings. A 10 -fold reduction in PG activity is consistent with previously described pCGN1436 transgenic tomato events (Redenbaugh et al., 1992). RNA from ripe tomatoes was separated on high and low molecular weight blots and probed for PG mRNA and siRNAs. Accumulation of PG mRNA was reduced $\approx 20$-fold in transgenic sibling tomatoes relative to nulls (Fig. 2A). Sense and antisense PG siRNAs were detected, consistent with an RNAi suppression mechanism (Fig. 2B). We also detected siRNAs from the coding region of the NptII cassette of the T-DNA. In contrast, we were unable to detect siRNAs from noncoding regions of the T-DNA or flanking genomic sequences (data not shown). A weak $1.5-\mathrm{kb}$ band corresponding to the correct size of NptII mRNA was detected by Northern blot (Fig. 2A) after a 3-d exposure. The long exposure and weakness of the bands indicate that the level of NptII mRNA was low, consistent with the presences of siRNAs. The majority of the siRNAs from the coding regions belong to the 21 -nt size class, although lower abundant 24-nt siRNAs were also observed. The decrease in PG message correlates with the reduction in $\mathrm{PG}$ enzyme activity levels, and the presence of 21-nt siRNAs correlates with the decrease in PG message.

\section{Discussion}

$P G$ gene suppression is the result of RNAi-mediated pathways. We found transgenic siblings of the commercial Flavr Savr tomato event contain two linked T-DNAs; however, unlike the eight right-border linkage events described by Sanders and Hiatt (2005), these plants contain T-DNAs linked at the left borders. While this configuration results in inverted $P G$ repeats, the promoter orientation would not be expected to generate a dsRNA precursor. Previous reports show using a single copy of an antisense transgene can trigger RNAi (Fagard and Vaucheret, 2000; Stam et al., 1997, 2000; Watson et al., 2005), and there are cases where cassettes lacking promoters induce gene silencing in plants with T-DNAs linked the same way we show here (Fagard and Vaucheret, 2000; Stam et al., 1997, 2000; Watson et al., 2005). Despite the unexpected difference in T-DNA linkage orientation, we observed an abundance of 21-nt siRNAs associated with sequence-specific mRNA degradation and translational repression (Brodersen and Voinnet, 2006; Fagard and Vaucheret, 2000; Hamilton et al., 2002; Watson et al., 2005). The 24-nt class, associated with transcriptional repression (Brodersen and Voinnet, 2006; Fagard and Vaucheret, 2000; Hamilton et al., 2002; Watson et al., 2005), was less abundant and some PG mRNA was still detectable in Flavr Savr tomatoes. If suppression of PG activity was due to sequestration from translation by binding to antisense RNA (Metzlaff et al., 1997), the accumulation of sense PG transcript should be unaffected and antisense transcripts would be detected. In contrast, if suppression were due to transcriptional or post-transcriptional gene silencing, accumulation of the message would be reduced, suggesting transgene-associated suppression was not due to repression of endogenous PG mRNA transcription but to RNAi (Brodersen and Voinnet, 2006). It is interesting that siRNAs were detected for PGAS and NptII, but not for any other regions of the T-DNA. This suggests that suppression requires transcription from the associated $P G$ and NptII promoters. Using flanking sequence as a query in searches with BLAST, we identified a region 1197-bp downstream of the T-DNA insert containing a putative cytochrome P450 gene (Fig. 1B). Potential promoter or enhancer elements associated with this gene may be associated with transcription across the T-DNA locus and could be responsible for contributing to the initial production of a dsRNA for the coding regions, thus resulting in the production of the siRNAs. This would also explain why we could not detect siRNAs from noncoding regions of the T-DNAs. The previously analyzed events have the potential to form PG dsRNA directly, in an RNA-dependent RNA polymerase (RdRP)independent mechanism via readthrough transcription from one $P G$ cassette in one T-DNA into the other $P G$ cassette in the other T-DNA (Sanders and Hiatt, 2005). It has been shown that there are several overlapping, and potentially hierarchical, mechanisms and pathways involved in plant gene silencing (Deleris et al., 2006). Without direct evidence of transcription across the transgene, we hypothesize an RdRP-dependent mechanism may be a contributor to enhancing siRNA production and gene silencing (Wassenegger and Krczal, 2006) compared with a dsRNA formed directly via readthrough transcription. It has been demonstrated repeatedly that expression of an antisense cDNA alone does not result in gene silencing as efficiently as expressing a dsRNA (Stam et al., 1997, 2000; Watson et al., 2005). Thus, the silencing mechanism of the Flavr Savr events first reported by Sanders and Hiatt (2005) might not be the only mechanism responsible for silencing in the commercial Flavr Savr tomato, event (CR3-1436-613:@.12/CF5013).PG gene suppression was achieved using an antisense expression cassette of the endogenous $(P G)$ 
A
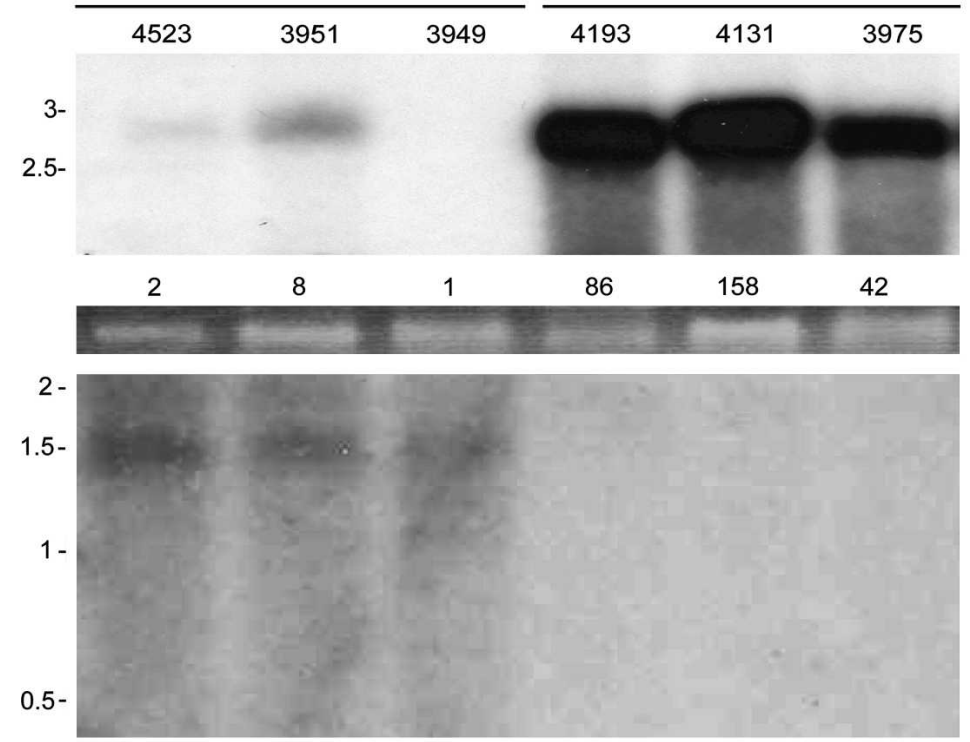

B

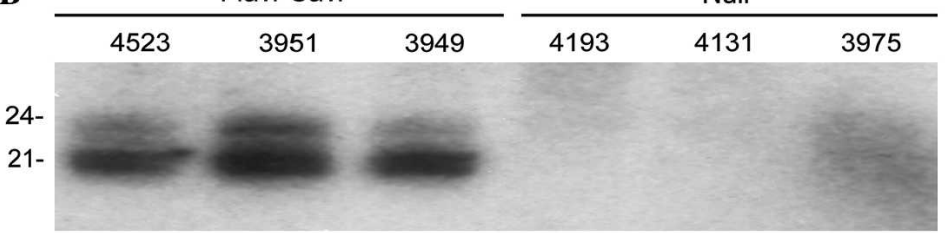

24-

21-

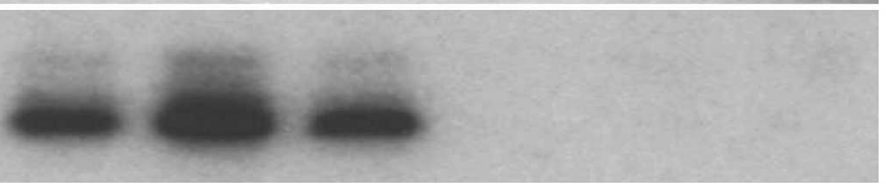

24-

21-

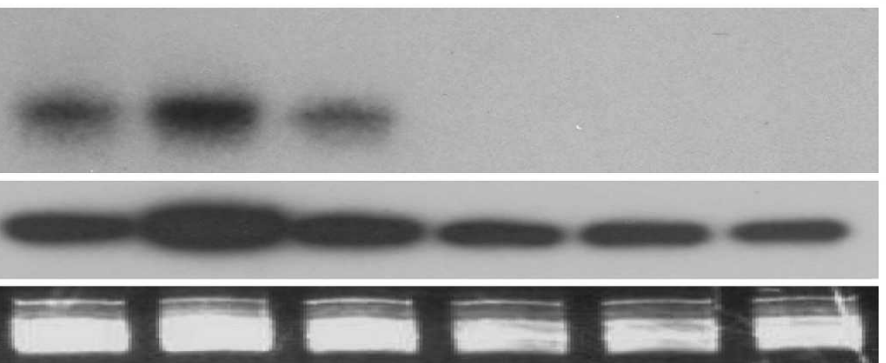

PG-s

NptII

$\operatorname{miR} 159$

$5 S$ rRNA/tRNA

Fig. 2. Suppression of PG correlates with accumulation of associated PG siRNAs. (A) Accumulation of PG mRNA in ripe transgenic $(4523,3951,3949)$ and null $(4193,4131,3975)$ sibling tomatoes. Relative accumulation (R.A.) of PG mRNA as determined by PhosphorImager quantitation is shown above the ethidium bromide-stained rRNA. Faint accumulation of NptII mRNA in ripe transgenic (4523, 3951, 3949) tomatoes and not in null $(4193,4131,3975)$ sibling tomatoes. (B) RNA blot analysis of 21- to 24-nt siRNAs associated with PG and NptII coding regions. 5S rRNA/tRNA bands and miR159 are shown as loading controls.

gene resulting in an RNAi-mediated pathway and yielding the phenotype of the Flavr Savr tomato. This research supports the need for precise characterization of gene insertion structures in relationship to understanding the mechanisms of gene suppression.

\section{Literature Cited}

Allen, E., Z. Xie, A.M. Gustafson, and J.C. Carrington. 2005. MicroRNA-directed phasing during transacting siRNA biogenesis in plants. Cell 121:207-221.

Brodersen, P. and O. Voinnet. 2006. The diversity of RNA silencing pathways in plants. Trends Genet. 22:268-280.

Deleris, A., J. Gallego-Bartolome, J. Bao, K.D. Kasschau, J.C. Carrington, and O. Voinnet. 2006. Hierarchical action and inhibition of plant Dicer-like proteins in antiviral defense. Science 313(5783):68-71.

Fagard, M. and H. Vaucheret. 2000. (Trans)gene silencing in plants: how many mechanisms? Annu. Rev. Plant Physiol. Plant Mol. Biol. 51:167-194.

Hamilton, A., O. Voinnet, L. Chappell, and D. Baulcombe. 2002. Two classes of short interfering RNA in RNA silencing. EMBO J. 21:4671-4679.

Llave, C., K.D. Kasschau, M.A. Rector, and J.C. Carrington. 2002a. Endogenous and silencingassociated small RNAs in plants. Plant Cell 14:1605-1619.

Llave, C., Z. Xie, K.D. Kasschau, and J.C. Carrington. 2002b. Cleavage of Scarecrow-like mRNA targets directed by a class of Arabidopsis miRNA. Science 297:2053-2056.

Metzlaff, M., M. O'Dell, P.D. Cluster, and R.B. Flavell. 1997. RNA-mediated RNA degradation and chalcone synthase A silencing in petunia. Cell 88:845-854.

Redenbaugh, K., W.R. Hiatt, B. Martineau, K. Kramer, S. Sheehy, R.A. Sanders, C. Houck, and D. Emlay. 1992. Safety assessment of genetically engineered fruits and vegetables: a case study of the Flavr Savr ${ }^{\mathrm{TM}}$ tomato. CRC Press, Inc., Boca Raton, FL.

Sanders, R.A. and W. Hiatt. 2005. Tomato transgene structure and silencing. Nat. Biotechnol. 23(3):287-289.

Sheehy, R.E., M. Kramer, and W.R. Hiatt. 1988. Reduction of polygalacturonase activity in tomato fruit by antisense RNA. Proc. Natl. Acad. Sci. USA 85:8805-8809.

Stam, M., R. de Bruin, S. Kenter, R.A.L. van der Hoorn, R. van Blokland, J.N.M. Mol, and J.M. Kooter. 1997. Post-transcriptional silencing of chalcone synthase in Petunia by inverted transgene repeats. Plant J. 12:63-82.

Stam, M., R. de Bruin, R. van Blokland, R.A. van der Hoorn, J.N.M. Mol, and J.M. Kooter. 2000. Distinct features of post-transcriptional gene silencing by antisense transgenes in single copy and inverted T-DNA repeat loci. Plant J. 21:27-42.

Wassenegger, M. and G. Krczal. 2006. Nomenclature and functions of RNA-directed RNA polymerases. Trends Plant Sci. 11:142-151.

Watson, J.M., A.F. Fusaro, M. Wang, and P.M. Waterhouse. 2005. RNA silencing platforms in plants. FEBS Lett. 579:5982-5987. 